\title{
Effects of $J_{2}$ Perturbation on Geometrical Relative Motion
}

\author{
W. A. Rahoma ${ }^{1,2^{*}}$ and F. Deleflie ${ }^{2,3}$ \\ 'Department of Astronomy and Space Science, Faculty of Science, Cairo University, 12613, Egypt; \\ walid_rahoma@yahoo.com \\ 2Laboratoire d'Astronomie de Lille/Université de Lille, 1 impasse de l'Observatoire 59000 Lille, France \\ ${ }^{3}$ Observatoire de Paris, IMCCE, GRGS, 75, Paris, France
}

\begin{abstract}
By exploiting a direct geometrical approach, an exact and efficient analytic formulation of relative motion was presented. Using the orbital elements without imposing any particular conditions on the base or the target satellites trajectories, exact expressions for the relative motion are obtained in a closed form. This solution allows the parameterization of the relative motion manifold and offers new methods to study its geometrical and topological properties. The study is complete and it maintains a high degree of accuracy even in the presence of $J_{2}$ perturbations. It is adequate for long-term prediction of bounded relative orbits.
\end{abstract}

Keywords: Geometrical Approach, Relative Motion, Satellites Constellations

\section{Introduction}

Due to the importance of rendezvous and docking maneuvers application between spacecrafts in presence of primary perturbing body like Earth, the study of the relative motion had concerned by many studies in the recent decades. Many traditional analysis for relative motion under the assumptions of a circular reference orbit, linearized differential gravity field (small relative distance), and without perturbations such as oblateness effects of the central body, are presented.

The first use of the relative motion was by $\mathrm{Hill}^{7}$ in the late $19^{\text {th }}$ century who was studying the motion of the Moon. His goal was to construct a more mathematically sound means of developing tables of lunar motion, which, at the time, were based on practical astronomy rather than of mathematics in his words. The first aerospace applications were in the area of intercept and rendezvous mechanics during the late 1950's and continuing today. The intercept problem is one in which a chase vehicle is forced in such a way that its path intersects the path of a target point (which may be occupied by another vehicle) at a specified time. The rendezvous problem further insists that the relative velocity of the two spacecraft be driven to zero at the time of intersection so that a docking procedure or other such activities may be conducted.

This problem was studied by Clohessy and Wiltshire ${ }^{3}$ in the interest of developing a guidance scheme for the rendezvous problem assuming that the target vehicle was in a circular orbit. This target satellite was to be a control center issuing relative position and velocity data to the slave satellites, which then used an on-board propulsion system to carry out the rendezvous and docking maneuver. Kelly ${ }^{9}$ developed an optimal solution to the two impulse rendezvous problem using relative motion equations and also includes the effects of eccentric orbits and gravity perturbations. A nonlinear model of relative motion was also given, but an analytical solution was not developed and so required numerical integration to solve the problem.

Alfriend et al. ${ }^{1}$, Vadali et al. ${ }^{2}$ and Schaub ${ }^{12}$ approached the problem by linearizing the direction cosine matrix of the orientation of the target with respect to the reference satellite. 
Garrison et al. ${ }^{6}$ used true anomaly as the independent variable to obtain analytical expressions for relative motion near high-eccentricity orbits.

Condurache and Martinui ${ }^{4}$ studied the relative orbital motion between arbitrary Keplerian trajectories. A closedform vectorial solution to the nonlinear initial value problem that models this type of motion with respect to a noninertial reference frame is offered.

This paper aims to find an exact solution for the relative motion problem under the $J_{2}$ perturbations. This solution will be adequate for long-term prediction of bounded relative orbits. A method is established to obtain the relative motion between spacecraft by direct geometrical interpretation of Keplerian elements. Here, the relative satellite motion without any restriction as to the plane of the satellite orbits as well as their eccentricity and inclination is studied.

\section{The Classical Approach}

Classical mechanics gives the expression of the relative acceleration with respect to a non-inertial frame (see Figure 1) with an angular velocity $\vec{\omega}$ and an acceleration $\vec{a}_{r e f}$. Using previous notions, the equations of the relative motion in a rotating reference frame become:

$$
\ddot{\vec{\rho}}=\vec{a}_{\text {Sat }}-\vec{a}_{r e f}-2 \vec{\omega} \times \dot{\vec{\rho}}-\dot{\vec{\omega}} \times \vec{\rho}-\vec{\omega} \times(\vec{\omega} \times \dot{\vec{\rho}})
$$

where $\vec{a}_{r e f}$ is the absolute acceleration of the body and the dot stands for the derivatives with respect to time and $\times$ is the operator of cross product

These equations use three variables: (i) the relative position and its derivatives, (ii) the difference of accelerations between the two satellites, and (iii) the rotation of the reference frame, also with its derivatives. Relative position is the unknown, and the other two variables can

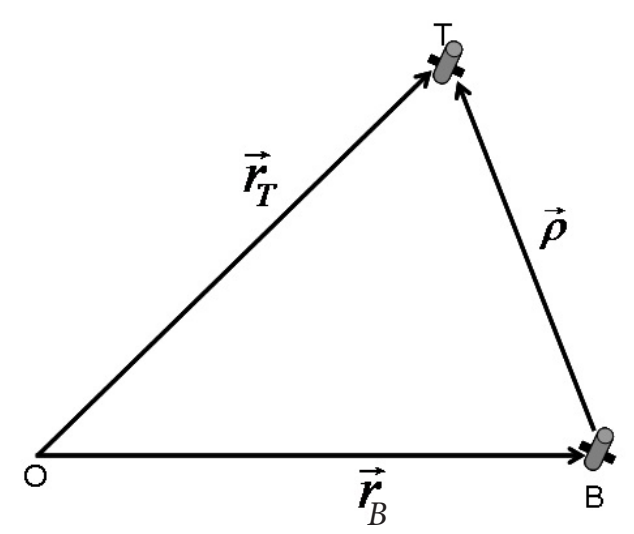

Figure 1. Geometry of the Relative Motion Problem. be modeled in different ways. Both of them depend on the reference orbit.

The problem of relative motion is best studied by using simple models to begin with. The easiest way, for modeling them, is to take a non-perturbed circular reference orbit. This choice leads to the well-known Hill or Clohessy-Wiltshire equations. If it is assumed that the reference satellite's orbit is circular, then $\vec{r}_{\text {ref }}$ is constant and the mean motion of the reference satellite $n=\sqrt{\mu / r_{r e f}^{3}}$, where $\mu$ is the product between the gravitational constant and the mass of the central body.

The resulting equations are referred to a well-known Hill's equations which integrate immediately to give:

$$
\begin{aligned}
\rho_{R}(t)= & \frac{\dot{\rho}_{R}\left(t_{0}\right)}{n} \sin n t-\left(2 \frac{\dot{\rho}_{T}\left(t_{0}\right)}{n}+3 \rho_{R}\left(t_{0}\right)\right) \cos n t \\
& +\left(2 \frac{\dot{\rho}_{T}\left(t_{0}\right)}{n}+4 \rho_{R}\left(t_{0}\right)\right), \\
\rho_{T}(t)= & \frac{2 \dot{\rho}_{R}\left(t_{0}\right)}{n} \cos n t+\left(4 \frac{\dot{\rho}_{T}\left(t_{0}\right)}{n}+6 \rho_{R}\left(t_{0}\right)\right) \sin n t \\
& +\left(-2 \frac{\dot{\rho}_{R}\left(t_{0}\right)}{n}+\rho_{R}\left(t_{0}\right)\right) \\
& -\left(3 \dot{\rho}_{T}\left(t_{0}\right)+6 n \rho_{R}\left(t_{0}\right)\right) t, \\
& \rho_{N}(t)=\rho_{N}\left(t_{0}\right) \cos n t+\frac{\dot{\rho}_{N}\left(t_{0}\right)}{n} \sin n t
\end{aligned}
$$

where, $\rho_{R}, \rho_{T}$ and $\rho_{N}$ refer to the radial, transferase and normal relative distance, respectively.

Lawden ${ }^{10}$ introduced the solution for the relative motion with eccentric non-perturbed reference orbit, using $f$ as the true anomaly, as

$$
\rho_{R}(t)=A \cos f+B e \sin f+C I_{2},
$$

$$
\begin{gathered}
\rho_{T}(t)=-A \sin f+B(1+e \cos f)+\frac{D-A \sin f}{1+e \cos f}+C I_{2}, \\
\rho_{N}(t)=\frac{1}{1+e \cos f}(E \cos f+F \sin f)
\end{gathered}
$$

with

$$
\begin{aligned}
& I_{1}=\sin f \int \frac{d f}{\sin ^{2} f(1+e \cos f)^{2}}, \\
& I_{2}=\frac{\cot f}{e(1+e \cos f)}+\frac{1+e \cos f}{e \sin f} I_{1}
\end{aligned}
$$


Fontdecaba ${ }^{5}$ addressed useful comments on this result, these are:

- Lawden gave a closed form of the solution, but he didn't give parameters $A, B, C, D, E, F$ as function of initial conditions.

- His solution is not convenient because of the integral $I_{1}$.

The nonlinear equations modeling relative motion in the two-body problem are given ${ }^{11}$ :

$$
\begin{gathered}
\ddot{\rho}_{R}-2 \dot{\theta} \ddot{\rho}_{T}-\dot{\theta}^{2} \rho_{R}-\ddot{\theta} \rho_{T}=-\frac{\mu\left(r_{\text {ref }}+\rho_{R}\right)}{\left[\left(r_{\text {ref }}+\rho_{R}\right)^{2}+\rho_{T}^{2}+\rho_{N}^{2}\right]^{\frac{3}{2}}} \\
+\frac{\mu}{r_{\text {ref }}^{2}-\frac{\partial \bar{V}}{\partial \rho_{R}},} \\
\ddot{\rho}_{T}+2 \dot{\theta} \ddot{\rho}_{R}-\dot{\theta}^{2} \rho_{T}-\ddot{\theta} \rho_{R}=-\frac{\mu \rho_{T}}{\left[\left(r_{r e f}+\rho_{R}\right)^{2}+\rho_{T}^{2}+\rho_{N}^{2}\right]^{\frac{3}{2}}} \\
-\frac{\partial \bar{V}}{\partial \rho_{T}}, \\
\quad-\frac{\mu \rho_{N}}{\left[\left(r_{r e f}+\rho_{R}\right)^{2}+\rho_{T}^{2}+\rho_{N}^{2}\right]^{\frac{3}{2}}} \\
-\frac{\partial \bar{V}}{\partial \rho_{N}}
\end{gathered}
$$

where, $\theta$ is the angle between the reduis vector of the referrence satellite and the $x$-axis of the inertial frame and $\bar{V}$ is the perturbed force.

Tschauner and $\mathrm{Hempel}^{8}$ changed the independent variable to the true anomaly, and scaled the relative position by the radius of the reference satellite

$$
\begin{gathered}
\rho_{R}^{\prime \prime}=\frac{3}{2} \frac{\rho_{T}^{2}+\rho_{N}^{2}-2 \rho_{R}^{2}}{1+e \cos f}+\mathrm{O}\left(|\rho|^{3}\right), \\
\rho_{T}^{\prime \prime}=\frac{3 \rho_{R} \rho_{T}}{1+e \cos f}+\mathrm{O}\left(|\rho|^{3}\right), \\
\rho_{N}^{\prime \prime}=\frac{3 \rho_{R} \rho_{N}}{1+e \cos f}+\mathrm{O}\left(|\rho|^{3}\right)
\end{gathered}
$$

If second- and higher-order terms are neglected then the resulting equations are known as the TschaunerHempel (TH) equations.

\section{Alternative Approach}

This section studies unperturbed and perturbed satellite relative motion, respectively, using direct geometrical approach. The results provide a complete analytic solution of satellite relative motion for formation flying and constellation design. It is useful to distinguish the variables used to represent the differential orbital elements. The Keplerian orbit is commonly specified by the classical orbital elements for state representations in space. The six orbital element sets are $[a, e, i, \omega, \Omega, f]$ where as usual $a$ is the semi-major axis, $e$ is the eccentricity, $i$ is the inclination, $\omega$ is the argument of the periapsis, $\Omega$ is the longitude of ascending node, $f$ is the true anomaly.

In the subsequent developments the adopted reference frame $(\mathcal{F})$ is an equatorial system with the positive $X$-axis towards the node of the orbit of base satellite, Z-axis towards the north pole of the equator of the primary, and the $Y$-axis completing a right handed system.

The position vectors of the base, $\vec{r}_{B}$, and target, $\vec{r}_{T}$, satellites can be written as the vector components in $\mathcal{F}$ using Figure 2, as:

$$
\begin{gathered}
\vec{r}_{B}=r_{B}\left(\begin{array}{c}
\cos u_{B} \\
\cos i_{B} \sin u_{B} \\
\sin i_{B} \sin u_{B}
\end{array}\right), \\
\vec{r}_{T}=r_{T}\left(\begin{array}{c}
\cos \Delta \Omega \cos u_{T}-\cos i_{T} \sin \Delta \Omega \sin u_{T} \\
\sin \Delta \Omega \cos u_{T}+\cos i_{T} \cos \Delta \Omega \sin u_{T} \\
\sin i_{T} \sin u_{T}
\end{array}\right),
\end{gathered}
$$

where, $r_{s}, s=B, T$ are the modulus of vectors $\vec{r}_{s}$ and $u_{s}=f_{s}+\omega_{s}$, usually known as the argument of latitude, and the subscripts $B, T$ refer to the base and target satellite, respectively, and $\Delta \Omega=\Omega_{T}-\Omega_{B}$, is the difference of the nodal longitudes.

The relative position vector $\vec{r}$ of the target satellite in base satellite centered frame, $\mathcal{F}$, are derived by vector subtraction of the position vectors from Eq. (5) and Eq. (6):

$$
\begin{aligned}
& \vec{r}_{T}=\left(\begin{array}{l}
x \\
y \\
z
\end{array}\right) \\
& =\left(\begin{array}{l}
r_{T}\left(\cos \Delta \Omega \cos u_{T}-\cos i_{T} \sin \Delta \Omega \sin u_{T}\right) \\
r_{T}\left(\sin \Delta \Omega \cos u_{T}+\cos i_{T} \cos \Delta \Omega \sin u_{T}\right) \\
r_{T} \sin i_{T} \sin u_{T}
\end{array}\right. \\
& \left.\begin{array}{l}
-r_{B} \cos u_{B} \\
-r_{B} \cos i_{B} \sin u_{B} \\
-r_{B} \sin i_{B} \sin u_{B}
\end{array}\right) \text {, }
\end{aligned}
$$




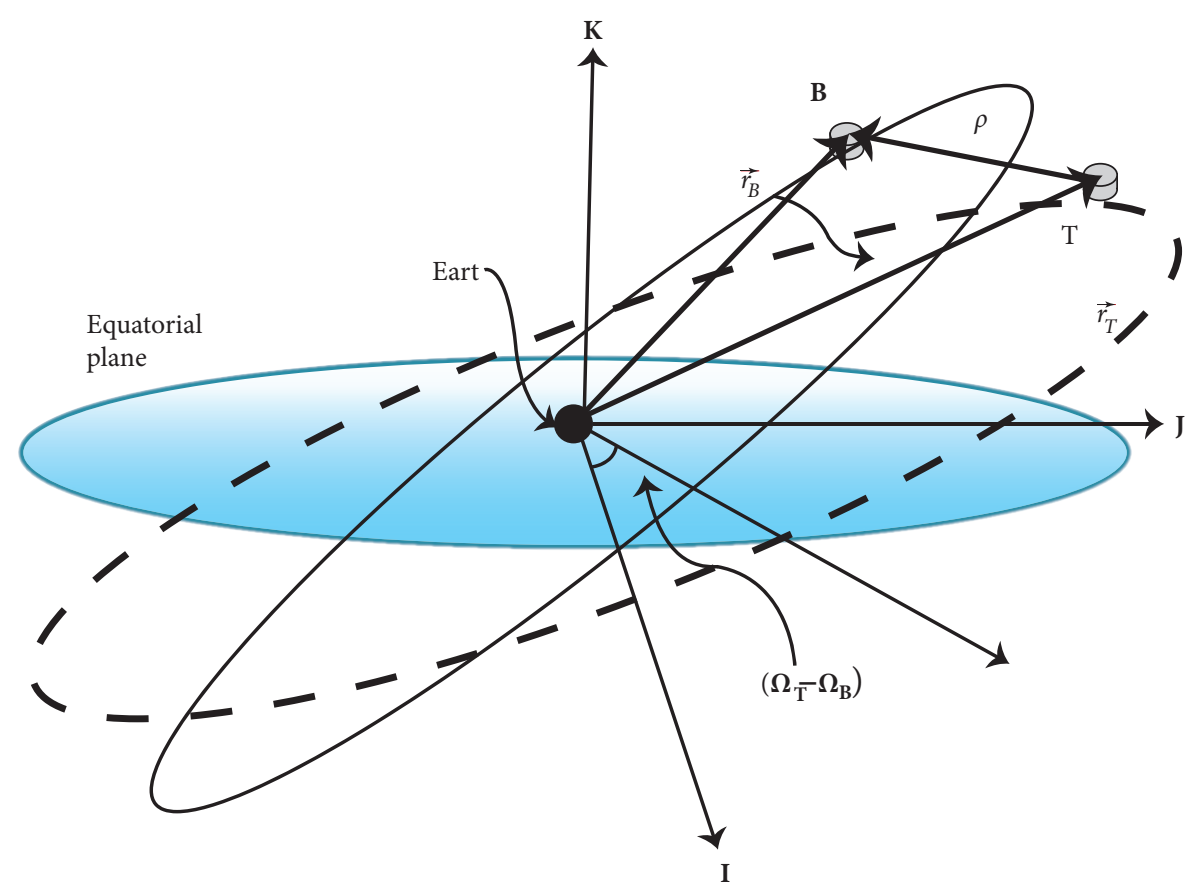

Figure 2. Relation between the relative distance and the orbital elements of two satellites with radius vectors $\vec{r}_{B}$ and $\vec{r}_{T}$.

The relative velocity vector $\vec{V}$ is obtained by taking the time derivatives of Eq. (7):

$$
\vec{V}=\left(\begin{array}{c}
\dot{x} \\
\dot{y} \\
\dot{z}
\end{array}\right)
$$

with

$$
\begin{aligned}
\dot{x}= & \sqrt{\mu}\left\{\frac{1}{\sqrt{a_{B}\left(1-e_{B}^{2}\right)}}\left[\sin u_{B}+e_{B} \sin \omega_{B}\right]\right. \\
& -\frac{1}{\sqrt{a_{T}\left(1-e_{T}^{2}\right)}}\left[\cos i_{T} \sin \Delta \Omega\left(\cos u_{T}+e_{T} \cos \omega_{T}\right)\right. \\
& \left.\left.+\cos \Delta \Omega\left(\sin u_{T}+e_{T} \sin \omega_{T}\right)\right]\right\}, \\
\dot{y}= & \sqrt{u}\left\{\frac{\cos i_{B}}{\sqrt{a_{B}\left(1-e_{B}^{2}\right)}}\left[\sin u_{B}+e_{B} \sin \omega_{B}\right]\right. \\
& -\frac{1}{\sqrt{a_{T}\left(1-e_{T}^{2}\right)}}\left[\cos i_{T} \cos \Delta \Omega\left(\cos u_{T}+e_{T} \cos \omega_{T}\right)\right. \\
& \left.\left.-\sin \Delta \Omega\left(\sin u_{T}+e_{T} \sin \omega_{T}\right)\right]\right\},
\end{aligned}
$$

For unperturbed motion, using the fact that $r_{s}=\frac{a_{s}\left(1-e_{s}^{2}\right)}{1+e_{s} \cos f_{s}}$.

The equations (7) and (8) are the exact analytic solution for satellite relative motions. The only assumption is that no perturbations are acting on the satellites.

\section{Effects of $\mathrm{J}_{2}$ on the Relative Distance}

In this section we will introduce a more accurate model. We will incorporate the Earth asphericity due to $J_{2}$ zonal harmonics. $J_{2}$ term is assumed to be of first. The gravitational potential including $J_{2}$ oblateness effects is given by

$$
\begin{aligned}
V(r, \varphi)=-\frac{\mu}{r}\left[1+J_{2}\left(\frac{R_{\oplus}}{r}\right)^{2} P_{2}(\sin \varphi)\right], & \\
J_{2} & =1.08269 \times 10^{-3}
\end{aligned}
$$


where, $\mu=G M_{\oplus}$, with $G$ is the universal gravitation constant, and $M_{\oplus}$ is the mass of the Earth. The quantities $r$ and $\varphi$ denote the radial distance and latitude of the satellite, respectively; $R_{\oplus}$ is the radius of the Earth, and $P_{2}(\sin \varphi)$ is the second Legendre polynomial.

The effects on the orbital elements due to the first order in $J_{2}$, are classified as secular growth, short-periodic, and long-periodic perturbations. If the study of the change of orbital elements is limited to that due to the first-order secular component, it can be shown that orbital elements $a, e$, and $i$ can be considered constant and the elements $\Omega$, $\omega$, and $f$ show secular growth.

So the following results are obtained from secular growth coming form $\mathrm{J}_{2}$ term

$$
\begin{aligned}
& \dot{a}=\dot{e}=\dot{i}=0 \\
& \dot{\Omega}=-J_{2} \frac{3 R_{\oplus}^{2} \pi \cos i_{0}}{2 a_{0}^{2}\left(1-e_{0}^{2}\right)^{2}}, \\
& \dot{\omega}=J_{2} \frac{3 R_{\oplus}^{2} \pi}{4 a_{0}^{2}\left(1-e_{0}^{2}\right)^{2}}\left(5 \cos ^{2} i_{0}-1\right), \\
& \dot{f}=J_{2} \frac{3 R_{\oplus}^{2} \pi \sin ^{2} i_{0}}{4 a_{0}^{2}\left(1-e_{0}^{2}\right)^{2}}
\end{aligned}
$$

In what follows we treated the considered modifications on the unperturbed model

$$
\vec{r}=\vec{r}^{0}+J_{2}\left(\begin{array}{c}
x^{1} \\
y^{1} \\
z^{1}
\end{array}\right)
$$

with

$$
\begin{aligned}
x^{1}= & -r_{T}^{0} \Delta \Omega^{1}\left(\cos u_{T}^{0} \sin \Delta \Omega^{0}+\sin u_{T}^{0} \cos \Delta \Omega^{0} \cos i_{T}^{0}\right) \\
& -r_{T}^{0} u_{T}^{1}\left(\sin u_{T}^{0} \cos \Delta \Omega^{0}+\cos u_{T}^{0} \sin \Delta \Omega^{0} \cos i_{T}^{0}\right) \\
& +r_{T}^{1}\left(\cos u_{T}^{0} \cos \Delta \Omega^{0}-\sin u_{T}^{0} \sin \Delta \Omega^{0} \cos i_{T}^{0}\right) \\
& -r_{B}^{1} \cos u_{B}^{0}+r_{B}^{0} u_{B}^{1} \sin u_{B}^{0}, \\
y^{1}= & -r_{T}^{0} \Delta \Omega^{1}\left(\cos u_{T}^{0} \cos \Delta \Omega^{0}-\sin u_{T}^{0} \sin \Delta \Omega^{0} \cos i_{T}^{0}\right) \\
& +r_{T}^{0} u_{T}^{1}\left(\cos u_{T}^{0} \cos \Delta \Omega^{0}+\cos i_{T}^{0}-\sin u_{T}^{0} \sin \Delta \Omega^{0}\right) \\
& +r_{T}^{1}\left(\cos u_{T}^{0} \sin \Delta \Omega^{0}+\sin u_{T}^{0} \cos \Delta \Omega^{0} \cos i_{T}^{0}\right) \\
& +\cos i_{B}^{0}\left(-r_{B}^{1} \cos u_{B}^{0}+r_{B}^{0} u_{B}^{1} \sin u_{B}^{0}\right),
\end{aligned}
$$

$$
\begin{aligned}
z^{1}= & \left(r_{T}^{0} u_{T}^{1} \cos u_{T}^{0}+r_{T}^{1} \sin u_{T}^{0}\right) \sin i_{T}^{0} \\
& -\left(r_{B}^{0} u_{B}^{1} \cos u_{B}^{0}+r_{B}^{1} \sin u_{B}^{0}\right) \sin i_{B}^{0}
\end{aligned}
$$

Using the superscript 0,1 for the unperturbed and perturbed case respectively, and

$$
\dot{\vec{r}}=\dot{\vec{r}}^{0}+J_{2}\left(\begin{array}{c}
\dot{x}^{1} \\
\dot{y}^{1} \\
\dot{z}^{1}
\end{array}\right)
$$

with

$$
\begin{aligned}
\dot{x}^{1}= & \sqrt{\mu}\left\{\frac{1}{\sqrt{a_{B}\left(1-e_{B}^{2}\right)}}\left[-u_{B}^{1} \cos u_{B}^{0}-\omega_{B}^{1} e_{B} \cos \omega_{B}\right]\right. \\
& -\frac{1}{\sqrt{a_{T}\left(1-e_{T}^{2}\right)}}\left[\Delta \Omega ^ { 1 } \left(\left(\cos \Delta \Omega^{0} \cos u_{T}^{0} \cos i_{T}^{0}\right.\right.\right. \\
& \left.-\sin \Delta \Omega^{0} \sin u_{T}^{0}\right)+e_{T}^{0}\left(\cos \Delta \Omega^{0} \cos \omega_{T}^{0} \cos i_{T}^{0}\right. \\
& \left.\left.-\sin \Delta \Omega^{0} \sin \omega_{T}^{0}\right)\right)+u_{T}^{1}\left(\cos \Delta \Omega^{0} \cos u_{T}^{0}\right. \\
& \left.-\sin \Delta \Omega^{0} \sin u_{T}^{0} \cos i_{T}^{0}\right)+\omega_{T}^{1} e_{T}\left(\cos \Delta \Omega^{0} \cos \omega_{T}^{0}\right. \\
& \left.\left.\left.-\sin \Delta \Omega^{0} \sin \omega_{T}^{0} \cos i_{T}^{0}\right)\right]\right\},
\end{aligned}
$$

$$
\begin{aligned}
\dot{y}^{1}= & \sqrt{\mu}\left\{\frac{-\cos i_{B}^{0}}{\sqrt{a_{B}\left(1-e_{B}^{2}\right)}}\left[u_{B}^{1} \cos u_{B}^{0}+\omega_{B}^{1} e_{B} \cos \omega_{B}\right]\right. \\
& +\frac{1}{\sqrt{a_{T}\left(1-e_{T}^{2}\right.}}\left[\Delta \Omega ^ { 1 } \left(\left(\sin \Delta \Omega^{0} \cos u_{T}^{0} \cos i_{T}^{0}\right.\right.\right. \\
& \left.+\cos \Delta \Omega^{0} \sin u_{T}^{0}\right)+e_{T}^{0}\left(\sin \Delta \Omega^{0} \cos \omega_{T}^{0} \cos i_{T}^{0}\right. \\
& \left.\left.+\cos \Delta \Omega^{0} \sin \omega_{T}^{0}\right)\right)+u_{T}^{1}\left(\sin \Delta \Omega^{0} \cos u_{T}^{0}\right. \\
& \left.+\cos \Delta \Omega^{0} \sin u_{T}^{0} \cos i_{T}^{0}\right)+\omega_{T}^{1} e_{T}\left(\sin \Delta \Omega^{0} \cos \omega_{T}^{0}\right. \\
& \left.\left.\left.+\cos \Delta \Omega^{0} \sin \omega_{T}^{0} \cos i_{T}^{0}\right)\right]\right\},
\end{aligned}
$$$$
\dot{z}^{1}=\sqrt{\mu}\left\{\frac{-\sin i_{B}^{0}}{\sqrt{a_{B}\left(1-e_{B}^{2}\right)}}\left[u_{B}^{1} \sin u_{B}^{0}+\omega_{B}^{1} e_{B} \sin \omega_{B}\right]\right.
$$$$
\left.+\frac{\sin i_{T}^{0}}{\sqrt{a_{T}\left(1-e_{T}^{2}\right)}}\left[u_{T}^{1} \sin u_{T}^{0}+\omega_{T}^{1} e_{T} \sin \omega_{T}\right]\right\}
$$ 
Using that

$$
\begin{gathered}
\omega_{s}^{1}=\frac{3 R_{\oplus}^{2} \pi}{2 a_{s}^{2}\left(1-e_{s}^{2}\right)^{2}}\left(5 \cos ^{2} i_{s}-1\right), \\
r_{s}^{1}=-\frac{3 R_{\oplus}^{2} \pi e_{s} \sin ^{2} i_{s} \sin f_{s 0}}{4 a_{s}\left(1-e_{s}^{2}\right)\left(1+e_{s} \cos f_{s 0}\right)^{2}}, \\
u_{s}^{1}=\frac{3 R_{\oplus}^{2} \pi \cos ^{2} i_{s}}{2 a_{s}^{2}\left(1-e_{s}^{2}\right)^{2}} \\
\Delta \Omega_{s}^{1}=\frac{3 R_{\oplus}^{2} \pi \cos i_{T 0}}{2 a_{T 0}^{2}\left(1-e_{T 0}^{2}\right)^{2}}+\frac{3 R_{\oplus}^{2} \pi \cos i_{B 0}}{2 a_{B 0}^{2}\left(1-e_{B 0}^{2}\right)^{2}} \\
=\frac{3 R_{\oplus}^{2} \pi\left(\frac{-\cos i_{T}}{2}+\frac{\cos i_{B}}{a_{T}^{2}\left(1-e_{T}^{2}\right)^{2}}+\frac{a_{B}^{2}\left(1-e_{B}^{2}\right)^{2}}{2}\right.}{2}
\end{gathered}
$$

\section{Numerical Application}

An example given below illustrates the influence of $J_{2}$ perturbation on the relative motion between two satellites. The orbital elements of the satellites used in the exemplary calculations are given in Table 1.

The numerical simulation, coded on iterating solutions at each time step; is 30 minutes, runs over a period of 90 days with the orbit elements of the base and target satellite in Table 1.

\section{Conclusion}

We developed the analytic solution for satellite relative motion through a direct geometrical description using the usual orbital elements without and with $J_{2}$ perturbations.

\begin{tabular}{|c|c|c|c|c|c|c|}
\hline Time & & & & & & \\
\hline $\begin{array}{c}\text { From } \\
1 / 1 / 1950\end{array}$ & $a(m) E+07$ & e E - 03 & $\mathrm{I}($ Deg $) \mathrm{E}+02$ & $\Omega(\mathrm{Deg}) \mathrm{E}+03$ & $\omega($ Deg $) E+03$ & $f($ Deg $) E+03$ \\
\hline
\end{tabular}
The derivation of this approach is straightforward, and the resulting equations provide a complete analytic form of the relative motion avoiding the singularity solution.

Table 1. Orbital elements of two satellites Grace A and Grace B

\begin{tabular}{llllllll}
\hline Grace A & 21762.00 & 0.683816980297970 & 0.647260795750325 & 0.890144804417850 & 0.356879216666980 & 0.350246638846765 & 0.333023742099671
\end{tabular}

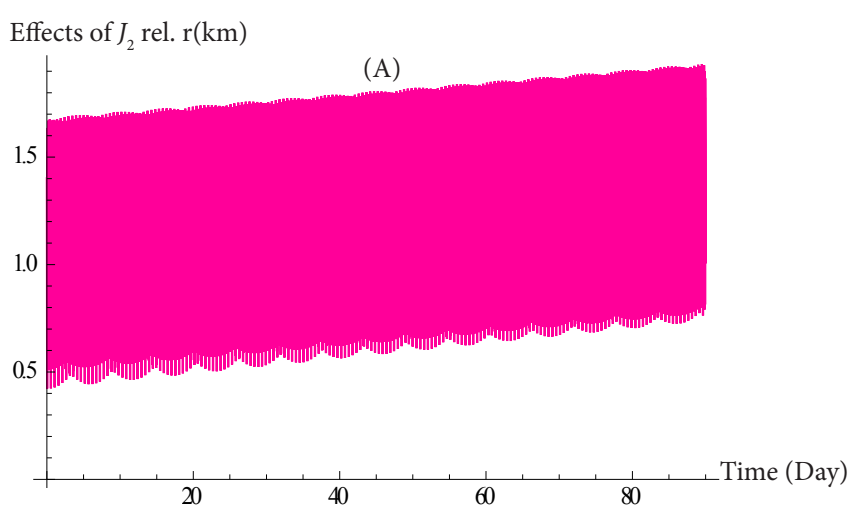

Effects of $J_{2} \mathrm{y}(\mathrm{km})$

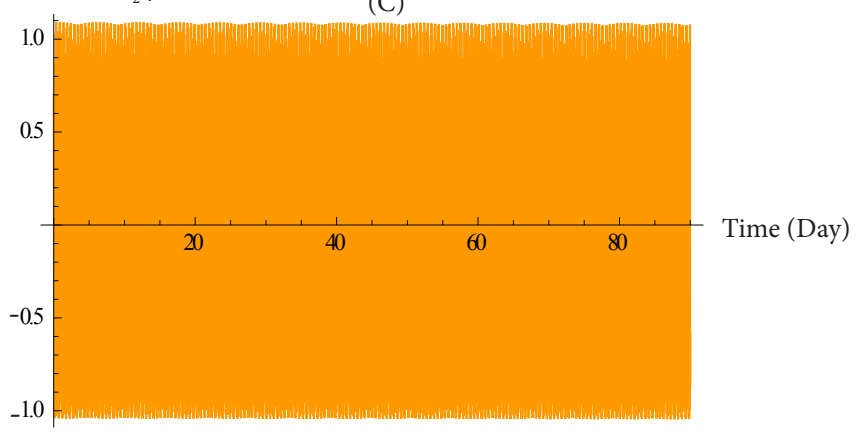

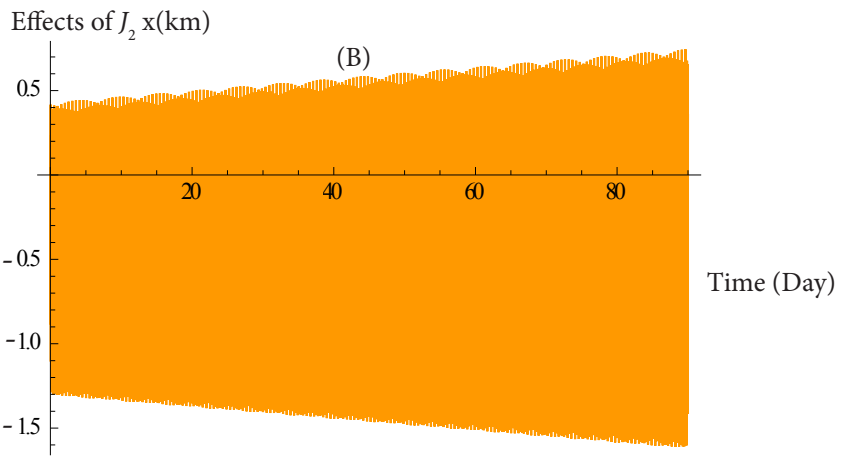

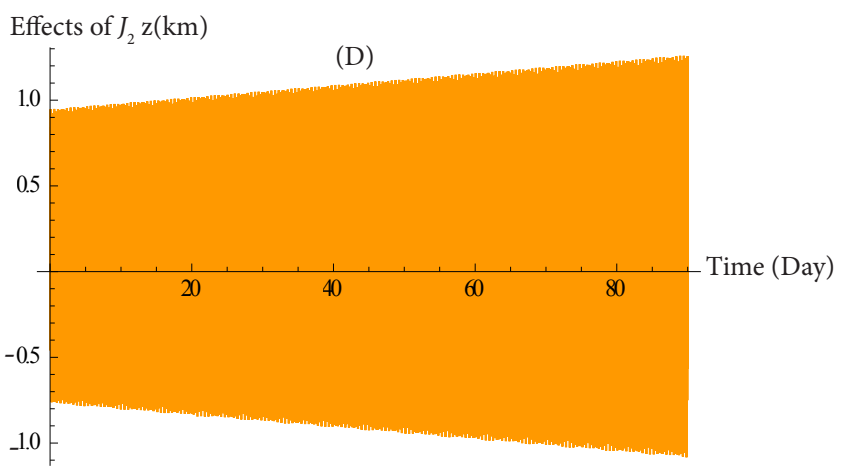

Figure 3. Values of differential perturbations in the relative distance of the satellites due to $J_{2}$ perturbations over 90 day. 

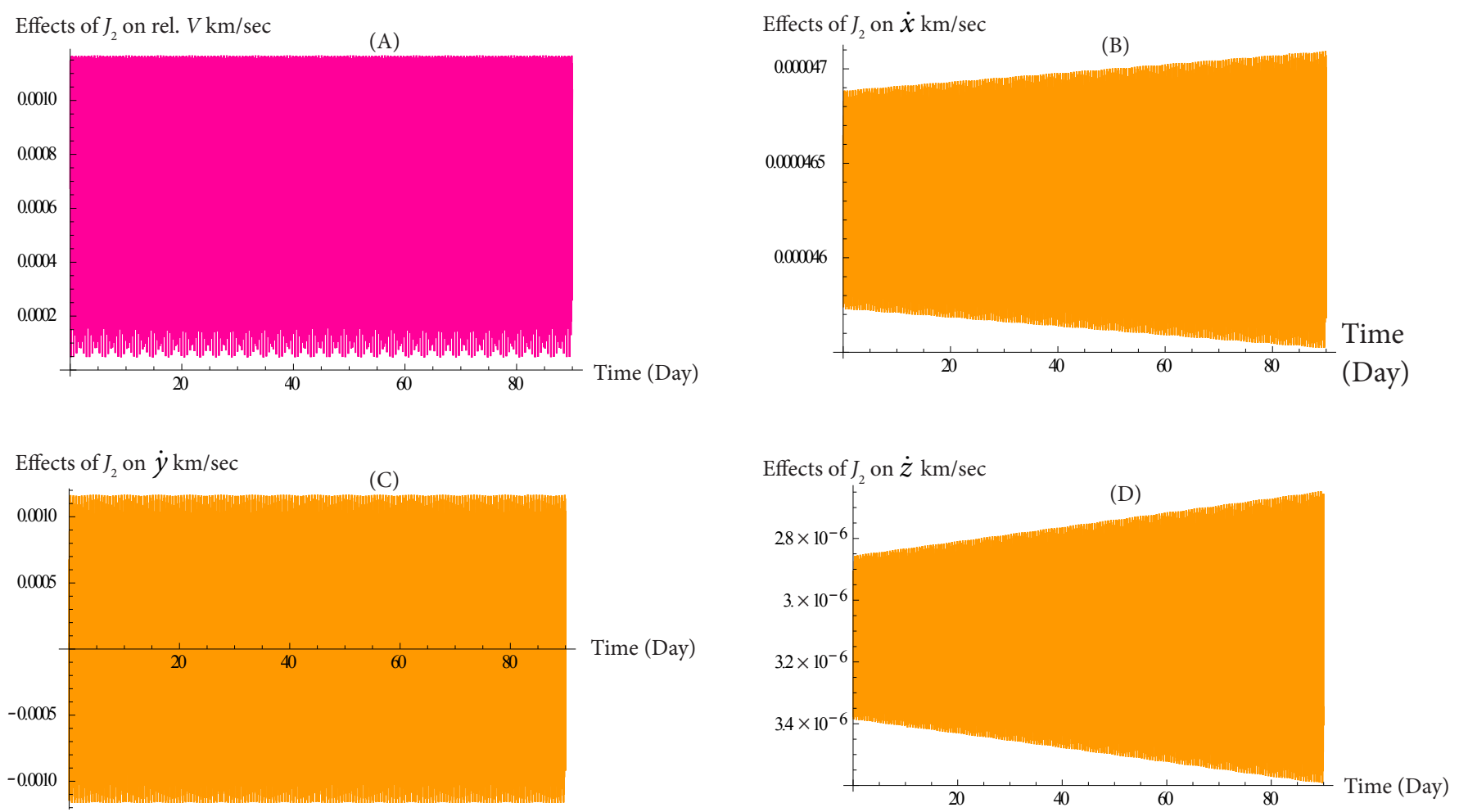

Figure 4. Values of differential perturbations in the relative velocity (rel. $V$ ) of the satellites due to $J_{2}$ perturbations over 90 day.

\section{References}

1. Alfriend KT, Schaub H, Gim DW. Gravitational perturbations, nonlinearity and circular orbit assumption effects on formation flying control strategies. Adv. Astronaut. Sci. 2000; 104:139-58.

2. Vadali SR, Vaddi SS, Alfriend KT. A new concept for controlling formation flying satellite constellations, Adv. Astronaut Sci. 2001; 108:1631-48.

3. Clohessy WH, Wiltshire RS. Terminal guidance system for satellite rendezvous. Journal of the Aerospace Sciences. 1960; 27:653-58.

4. Condurache D, Martinusi V. Exact solution to the relative orbital motion in eccentric orbits. Solar System Research. 2009; 43:41-52.

5. Fontdecaba J. Dynamics of formation flying applications to earth and universe observation, [Ph.D. Thesis], Observatoire de Paris EcoleDoctoraleAstronomie et Astrophyisiqued 'Ile de France; 2008.

6. Garrison JL, Gardner TG, Axelrad P. Relative motion in highly elliptical orbits, Adv Astronaut Sci. 1995; 89:1359-76.

7. Hill GW. Researches in the Lunar Theory. Am J Math. 1878; 1:5-26, 129-47, 245-60.

8. Tschauner JFA, Hempel PR. Rendezvous zueineminelliptischerbahnumlaufendenziel. Astronaut Acta. 1965; 11:104-9.

9. Kelly TJ. An analytical approach to the two-impulse optimal rendezvous problem. Advances in the Astronautical Sciences. 1994; 87:337-47.

10. Lawden DF. Optimal trajectories for space navigation, Butterworth and Co. (Publishers) Ltd; 1963.

11. Rahoma WA. Co-orbital motion with the earth as a central body, [Ph.D. Thesis], Cairo University, Egypt; 2009.

12. Schaub H. Relative orbit geometry through classical orbit element differences, J. Guid. Control Dynam, 2004; 27:839-48. 